\title{
Dynamic Spectrum Sensing By Channel Information Table in Cognitive Radios
}

\author{
M.K.Kaushik, Y. Yoganandam \\ (Department Of Electrical Engineering, BITS-Pilani, Hyderabad Campus, Hyderabad, India)
}

\begin{abstract}
The spectrum sensing problem has gained new aspects with cognitive radio and opportunistic spectrum access concepts. It is one of the most challenging issues in cognitive radio systems. In cognitive radio networks, the secondary (unlicensed) users need to find idle channels via spectrum sensing for their transmission. Adaptive spectrum sensing (ASS) is a promising technology in spectrum sensing with an admirable performance. In ASS, multiple secondary users individually sense the idle channels and send their decisions to the network center, and then the center will do a final decision. Spectrum sensing, which aims at detecting spectrum holes, is the precondition for the implementation of cognitive radio $(C R)$. In this work we formed an information table at MSC (main switching center) which consists of channel information and according to the requirement any primary or secondary user can fetch that channel and utilize without any interference. The experiment is conducted on static as well as adaptive environment to check the throughput, route delay of the conventional as well as proposed method.
\end{abstract}

Keywords: cognitive radio, cognitive radio network, MANets, spectrum sensing, wireless sensor networks

\section{Introduction}

RADIO spectrum is usually considered as a scarce resource while measurements show that the allocated spectrums are vastly under-utilized. The cognitive radio (CR), which has received a considerable attention, is designed for the secondary users to opportunistically access to the unused primary (licensed) spectrums without causing interference to the primary users. Ever since the 1920s, every wireless system has been required to have an exclusive license from the government in order not to interfere with other users of the radio spectrum. Today, with the emergence of new technologies which enable new wireless services, virtually all usable radio frequencies are already licensed to commercial operators and government entities. According to former U.S. Federal Communications Commission (FCC), spectrum is not utilized according to the data rates. On the other hand, not every channel in every band is in use all the time; even for premium frequencies below $3 \mathrm{GHz}$ in dense, most bands are quiet most of the time.

The FCC in the United States and the Ofcom in the United Kingdom, as well as regulatory bodies in other countries, have found that most of the precious, licensed radio frequency spectrum resources are inefficiently utilized. Cognitive radios will help commercial as well as military communication systems, by eliminating the need for comprehensive frequency planning. They will be capable of sensing the environment, making decisions on the types of signals present, learning patterns, and choosing the best method of transmitting the information either on their own or in a network- centric environment. They will be situationaware and capable of making decisions to ensure error-free and smooth transfer of bits between the users. Cognitive radios will be based on software defined radio (SDR) platforms and will try to understand not only what users want but also what the surrounding environment can provide. Hence spectrum sensing and utilization will be done according to the limits and channel requirements. Through the detection and utilization of the spectra that are assigned to the licensed users but standing idle at certain times, cognitive radio acts as a key enabler for spectrum sharing. Spectrum sensing, aiming at detecting spectrum holes (i.e., channels not used by any primary users), is the precondition for the implementation of cognitive radio. The Cognitive Radio (CR) nodes must constantly sense the spectrum in order to detect the presence of the Primary Radio (PR) nodes and use the spectrum holes without causing harmful interference to the PRs. Hence, sensing the spectrum in a reliable manner is of vital importance and constitutes a major challenge in CR networks. However, detection is compromised when a user experiences shadowing or fading effects or fails in an unknown way.

Lot of research is happening on spectrum sharing and utilization. In this work the focus is towards maintaining a spectrum information table at the main switching center and allocates the frequencies accordingly without any interference among the primary user and secondary user. As the motivation is towards combining computer networks and communications at one platform and looking for quality of service, applicationof routing protocol also plays main role. Once the optimized routing protocol is applied and spectrum information table is maintained properly the idle spectrums are assigned to secondary users without any interference. 


\section{Literature Survey}

Spectrum sensing is a key issue in cognitive radio systems. Single-user spectrum sensing may not be reliable due to many uncertainties such as fast fading or shadowing.Cognitive radio has received a lot of interest over the past few years as a promising technology that aims to alleviate the spectrum scarcity problem by allowing unlicensed (secondary) users to access spectrum that is allocated to licensed (primary) users under the condition of preserving the quality-of-service (QoS) of the licensed networks. In order to locate the nodes which are in seek of communication is first explored by routing protocol and then the information is sent to the information table and the communication happens in reliable fashion. Some of the optimized routing protocols which are in current research are mentioned.

Routing Protocols in Ad-Hoc networks have been classified in many ways [3], based on routing strategy and network structure. According to routing strategy, the routing protocols can be categorized as tabledriven and source initiated, while depending on the network structure these are classified as flat routing, hierarchical routing and geographic position assisted routing. It should be mentioned at this point that owing to the dynamic link topology, there are both unipath and multipath routing protocols. Most multipath routing protocols are built upon unipath routing protocols, hence we restrict our discussion to mostly unipath routing protocols.

A lot of the routing protocols mainly make use of the flooding technique for route discovery which we briefly mention as follows: A sender $\mathrm{S}$ broadcasts data packet $\mathrm{P}$ to all its neighbors and each node receiving $\mathrm{P}$ forwards $\mathrm{P}$ to its neighbors - In this way, packet $\mathrm{P}$ reaches the destination $\mathrm{D}$ provided a path from $\mathrm{S}$ to $\mathrm{D}$ exists. Node D does not forward the packet. We describe two routing protocols that make use of this technique, namely Dynamic Source Routing (DSR)[4] and Ad-hoc On-Demand Distance Vector Routing (AODV)[5]. DSR uses source routing instead of relying on the routing table at each intermediate device. DSR uses a combination of Route Request (RREQ), Route Reply (RREP) and Route Error (RERR) messages to establish a route from source to destination. In addition DSR also uses route caching to speed up route discovery. The main advantage of DSR is that a route to a destination is established only when it is required to, and a single route discovery often discovers routes to other destinations in the process. However, the main drawbacks of DSR are increased size of packet header with the length of the route, stale caches, etc. AODV is a reactive (on-demand) protocol which borrows the advantageous concepts from DSR and DSDV (Destination Sequenced Distance Vector Routing)(We mention this later) like on-demand route discovery and route maintenance, and the usage of node sequence numbers from DDSV. The actual working of AODV is beyond our scope. Reference [5] gives a good description. AODV is one of the most successful routing protocols in MANETs mainly because of its desirable features like Minimal Space Complexity and Maximum Bandwidth Utilization.

We now look at another widely deployed protocol called Destination Sequenced Distance Vector Routing Protocol (DSDV) [6]. DSDV is a proactive (means routes are independent of traffic pattern) protocol which is a modification of the conventional Bellman-Ford algorithm. The important feature of DSDV is the use of sequence numbers for the routing table entries. These sequence numbers are generated by the destination stations and routing tables at each node are synchronized by each node advertising its routing table information to its neighbors frequently. Forwarding decisions are made based on the sequence numbers. The main advantages of DSDV are that it guarantees loop-free paths and always maintains the best path to destination rather than multiple paths. However, routing table updates are costly, and there is no support for multi-path routing.

More recently, because of the rapid advances in Global Positioning Systems (GPS), Geographic Routing Protocols are becoming popular. One such protocol is the Geographic Distance Routing (GEDIR) [7]. In GEDIR, location of the destination node is assumed to be known, and each node knows the locations of its neighbors and forwards a packet to the neighbor closest to the destination. Many interesting advances have been made in Geographic Routing like Routing based on Virtual Co-ordinates [9], Routing without Location Information [8], etc.

In addition to Reactive and Proactive routing protocols, we also have Hybrid schemes like the Zone Routing Protocol (ZRP) [10] which proactively maintain state information for links within a short distance, say $\mathrm{d}$, from any given node (Intra-Zone Routing) and uses a route discovery protocol for determining routes to a nodes at a distance greater than d (Inter-Zone Routing).

Most protocols we have seen so far use some kind of flooding. There are few protocols like the Link Reversal Algorithm and Temporally-Ordered Routing Algorithm (TORA) which try to avoid/reduce flooding behavior. See [11] and [12] respectively for discussions. Routing Protocols which define the optimization criteria as a function of the energy consumption are called Power-Aware Routing Protocols [13]. The idea is to assign each link a weight which is a function of energy consumed when transmitting a packet on that link. The goal is to route through paths with minimum weight. Such protocols are usually built on top of existing protocols like DSR. 


\section{Design Approach:}

Since the mobile terminals are free to move, it is not guaranteed that any MT involved in a communication session will remain in the cell it once was during the lifetime of the session. When an MT goes over to another cell while a call proceeds, the call is passed to the new base station. This procedure is called handoff. The average number of handoffs during a session depends in the cell radii, mobility pattern of the mobile terminals and call duration.

Two thresholds are set for the handoff procedure. When the power level drops below the first threshold, the handoff procedure is triggered. As long as the power level remains above the second threshold, which is lower than the first one, the perception of the signal is enough for proper communication. In order to provide seamless communication, the received signal powers are monitored. The handoff must be completed before the signal level drops below the second threshold.

In case of a handoff, the call is transferred to the base station of which the received power level is highest. Therefore an MT, the initial and a final BS is involved in a handoff procedure. The transfer of preestablished communication links from one base station to another one takes time. It is possible that new resources are not available on the way to the new base station. Especially when the carried data rate cannot be determined beforehand, it may be difficult to allocate necessary resources on time. Hence, the gap between two threshold values should be chosen very carefully to allow the procedure enough time to be completed. Details and an overview of handoff strategies can be found in [14].

Another important issue is that the data should not be lost during the handoff. According to the properties and aims of the application and network, deployment of buffers may be necessary. If only multimedia content is transferred, the buffering may not be necessary. On the other hand, deployment of buffers is inevitable for handoffs for data transfer. The buffer deployment strategies and how the buffered data can be transferred to the MT is discussed in [13].

When the scope of the cellular networks is extended to include also multicasting, different considerations are necessary [14]. Buffering is also deployed in cases of multicasting data to many mobile terminals over heterogeneous data rates. The synchronization of data during handoffs is a very challenging problem. A possible solution to this problem is presented in [15].

\section{Mobility Management}

It is not enough to provide access to the mobile terminals or keep the communication alive despite the mobility. It is also of vital importance to keep track of the locations of the mobile terminals. Again no assumption about the location of the mobile terminal is logical. Hence, there should be a way of finding the mobile terminal in a reasonable time and at a reasonable cost. The efforts to locate the mobile terminals in the entire network and update of the location information are called Mobility Management.

There are mainly two fundamental operations in location management [16]. The first one is location update. Location update corresponds to reporting the location of the mobile terminal to its home location register, a database at the home of the mobile terminal. The second operation is paging. This operation corresponds to broadcasting the identity of a mobile terminal over a certain area. These two operations are inevitable for locating the mobile terminal. When a call is directed to a mobile terminal, the home location register is checked to find out the location of the mobile terminal. Then, the call is redirected to the actual location of the mobile terminal. When the call reaches the designated location, the mobile terminal is paged.

The location data stands most of the time for a group of cells, which is called a location area [17]. The location update is performed when a mobile terminal enters the location area. If the location area consists of only one single cell, the exact location is retrieved from the home location register and the paging cost is minimized. But this would lead to high location update costs and create lots of signaling traffic significantly. On the other hand, if the size of the location area is increased, the frequency of location updates decreases. However, paging has to be performed in more than one cell. Note that even if the location area consists of only one cell, paging should be performed.

There are three major different location update strategies. The update may be based on the time elapsed, the number of movements [16] or the distance traveled [17]. In any case, the location uncertainty introduced with location areas persists. Therefore, for each approach, the trade-off between the traffic generated and paging cost has different levels. It is also possible to optimize the location update procedure. In order to achieve this, a hierarchical structure is proposed in [18]. Here the information dissemination follows a hierarchical structure. So the amount of data flowing in the network is lowered.

Different ideas and approaches are proposed in the literature to optimize the mobility management. These considerations also take different levels of constraints and mobility models into account. The common aims, though, are minimizing the paging and communication load while keeping the location delay in acceptable amounts. Some related approaches can be found in [16-19]. 


\section{Channel Allocation Schemes}

The total amount of channels available for the cellular network can be assigned to cells in different ways. The process of assigning channels either to cells or to calls is called channel allocation. The schemes used differ in performance, flexibility and complexity. However, there is a tradeoff among these qualities, hence each scheme has advantages and disadvantages.

Channels can be distributed to cells statically, dynamically or in hybrid ways. The most commonly used scheme is to assign the channel sets statically to the cells. The cells may use the channels from the available set without any other constraint. This scheme performs well under the uniform traffic load over the cells. Its performance is also high in cases where the total traffic load is high.

In order to cope with situations where the traffic load differs from one cell to the other and where traffic load ratios do not change very much over the time, the cells may be borrowed to heavily loaded cells. If the channel set sizes are not equal, this corresponds to static borrowing. If the system has hot spots where the traffic intensity increases for short periods of time, then the channels may be lent by the lightly loaded neighboring cells to the temporarily highly loaded cell. Then the problem is solved at the cost of not being able to use the borrowed cells in a larger area than before.

It is also possible to set limits on the number of cells that can be borrowed in order to prevent starvation. In any case, a certain borrowing algorithm has to be deployed. The algorithm may choose channels from the richest cell. Another choice would be to borrow the first available channel in the neighborhood. It is also possible to return the used channels back to the donor as soon as a channel from the set of channels that belong to the cell that borrowed becomes available.

It is possible to assign the channels to the cells according to their needs. The channels are kept in a common set. The cells do not have their own channels. The assignment is done in call basis. In order to stick to the rules of frequency reuse, the determination of channels to be assigned has to be made carefully. The schemes that are based on the dynamic channel allocation are examined according to the way the assignment decision is performed.

The first scheme has a central decision element which decides on the channels that has to be assigned to calls. As it is the case for all centralized systems, the main drawback of this approach is that the central authority becomes the bottleneck of the system. So it is possible to distribute the decision to the base stations. The base stations collect local data from their vicinity and make their allocation decisions according to this. The resulting assignment is not optimal due to local character of the collected data. The results can be specialized and optimized for the one dimensional movement case.

Another possibility is to combine both approaches into one hybrid system. The entire channel spectrum is divided into two subsets. The first set is distributed to the cells statically. The remaining channels are assigned to cells according to the traffic load and call requests. Since the two systems are combined, any choice of the sub-strategies can be applied to the respective part of the scheme.

\section{System Architecture:}

To develop a simulation model and generalized architecture is designed as shown below,

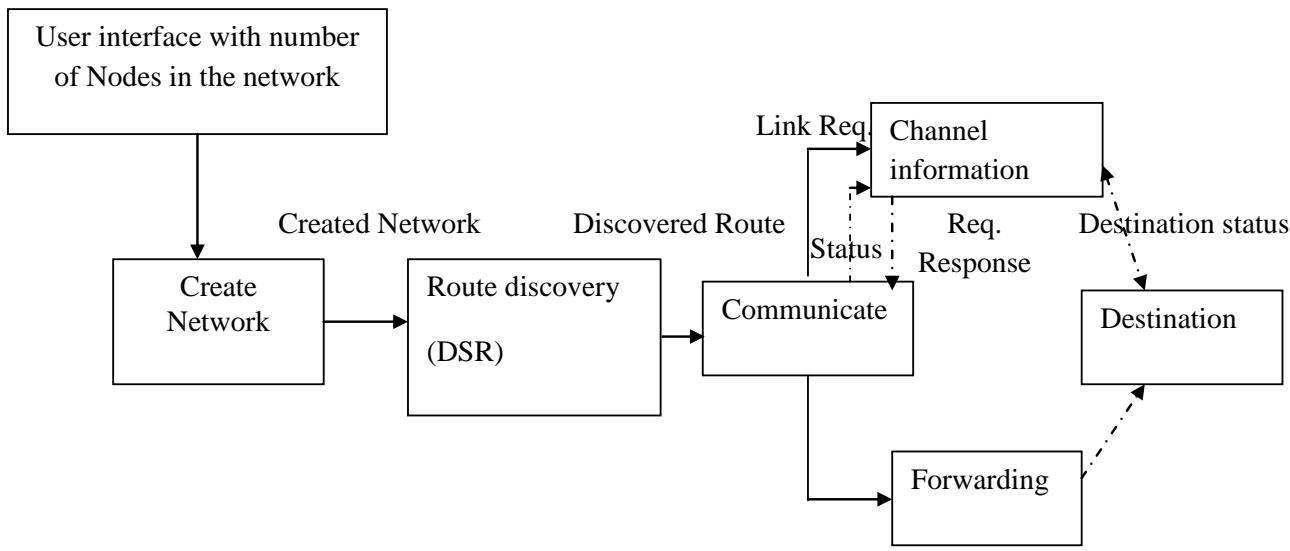

Figure1: System Architecture

The first group of experiments deals with the effect of the isolated parameters on the objective function. For all of these parameters, the relationship between the parameters supplied to the algorithm and the cost of the system is compared. Furthermore the effects of the design decisions are questioned. Their effect on the cost is compared with the cost of the system in hand. The comparison is made by comparing the costs under different arrival rates. 
The aim of the experiments in the second group was to observe the solutions of the adaptive algorithm in a large part of the parameter space. By doing so, the behavior of the spectrum sensing systems is presented.

The performance of the system under some unexpected increases in the call arrival rates is examined. The increase in the call arrival rates include calls generated by both mobility class users. The obtained results are presented graphically.

Lastly, the time complexity of the algorithm is estimated partially by experimental measurements and partially by analysis of the algorithms used in the solution technique. The results of this analysis are also presented in this section.

For the realization and evaluation of the suggested approach following matlab coding were developed,

1) Msc_mont.m: This function is developed for central monitoring of the channel allocation happening in the cells by the base stations for the generated request.

2) Data_ex.m: This function is developed for the data exchange between two nodes during uplink and downlink operation.

3) Comm.m: This function is developed for the selection of the communication process from given source to destination based on one-hop or two hop communication

4) Creat_ntw.m: This function is developed for the creation of random nodes and the network during the network creation.

5) Route.m: this function implements the routing protocol in route establishment in the cellular adhoc network. This function estimates the neighbor node, broadcast the packet from a source to destination and predict all possible paths for creation of possible routes for a given source to destination.

6) Main.m: this is the main unit of the implementation and integratesthe entire sub module for the realization of the functionality.

7) Node: this function realizes the node unit where the address matching is performed and if the destination match with the current node address then acknowledgment packet is generated else the packet is forwarded to the next neighbor cell.

8) Path23.m: this function realizes the path creation from a source to destination. it buffers the one hop neighbor until the destination is reached in uplink operation.

9) Sel_channel.m: This function selects the channel to be allocated from the IIT list based on the given source and destination in a cell.

The operational flow chart is as presented below

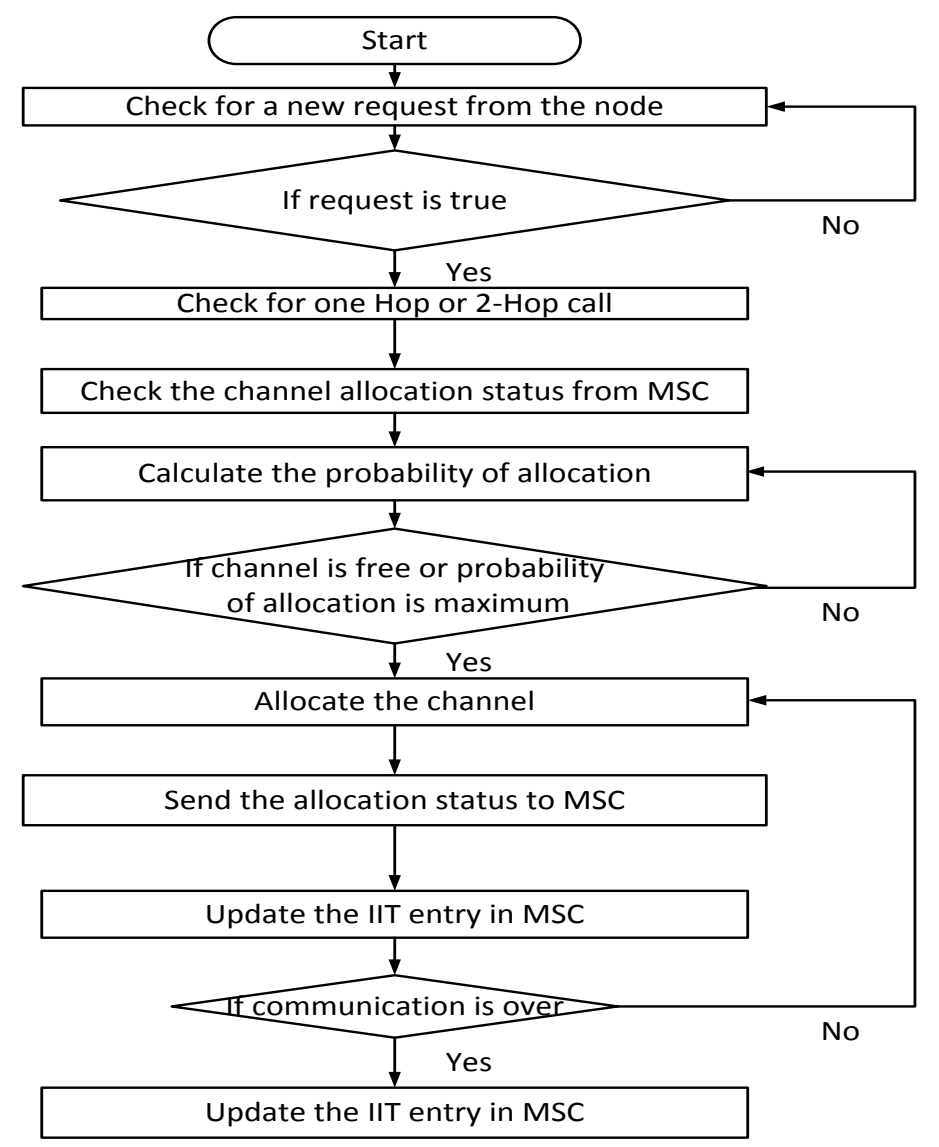

Figure 2: Operational flow chart

www.iosrjournals.org 


\section{Results}

The algorithm is simulated by two case studies viz., static and dynamic topology which is shown in figures 3 and 4 respectively. From the figures it's been observed that with the help of channel information table at main station the primary user and secondary users are allocated without any interference. Some of the parameters like route delay, throughput, route overhead are tested on two kind of topologies with and without table information at main station. It's clearly observed that a reliable path is obtained by dynamic routing protocol and throughput of the system is also maintained properly between licensed and unlicensed users

Case Study 1:Spectrum Sensing when Nodes in the Base Station Area are Static

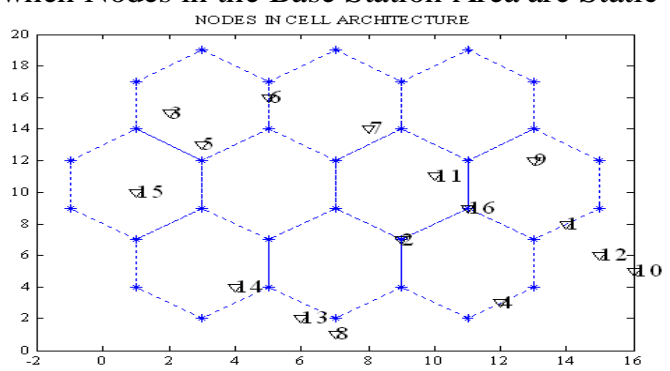

Figure 3Developed cellular architecture and node distribution for the simulation

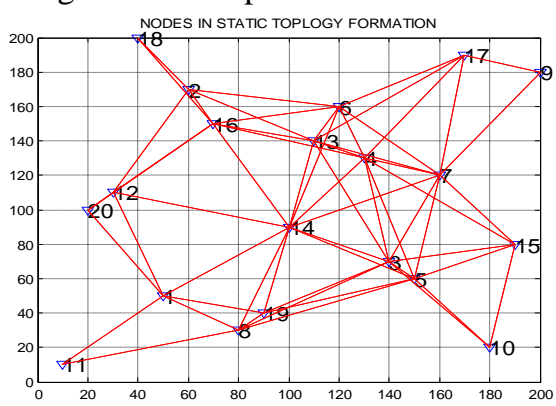

(a)

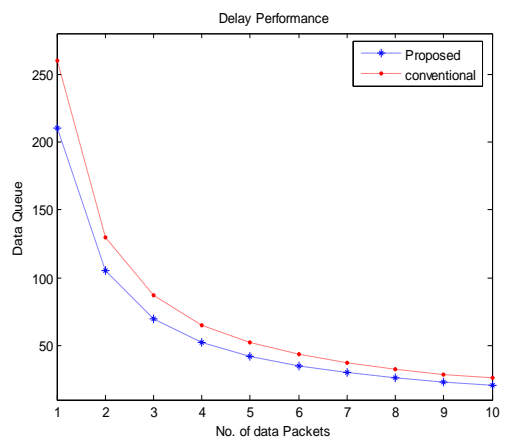

(c)

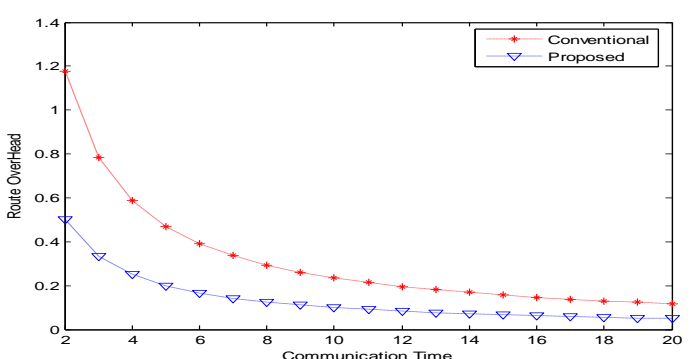

(e)

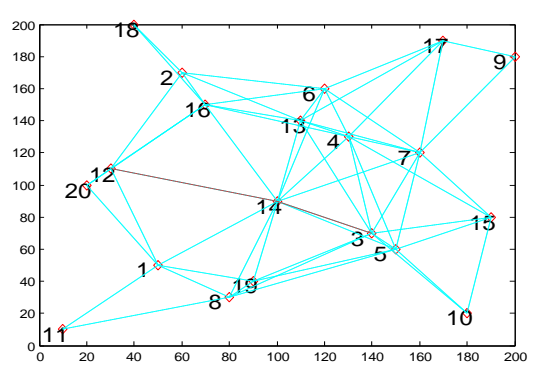

(b)

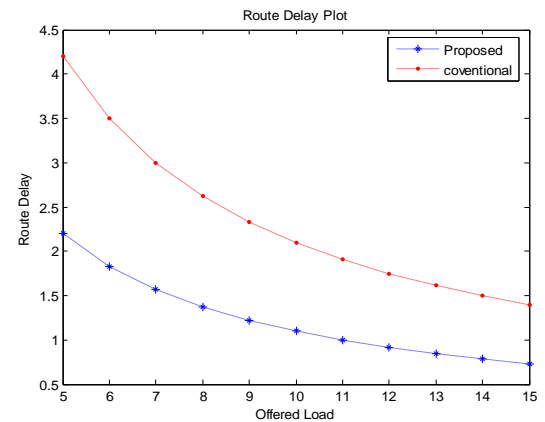

(d)

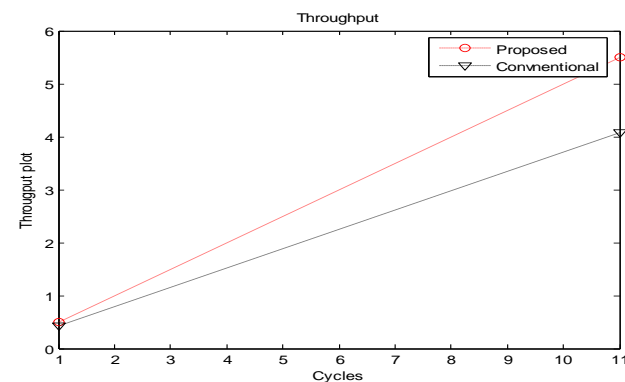

(f)

Figure 4Static topology (a) Nodes in topology (b) Reliable Route (c) Delay Performance (d) Route Delay Plot (e) Route overhead (f) Throughput 
Case Study 2:Dynamic Spectrum Sensing

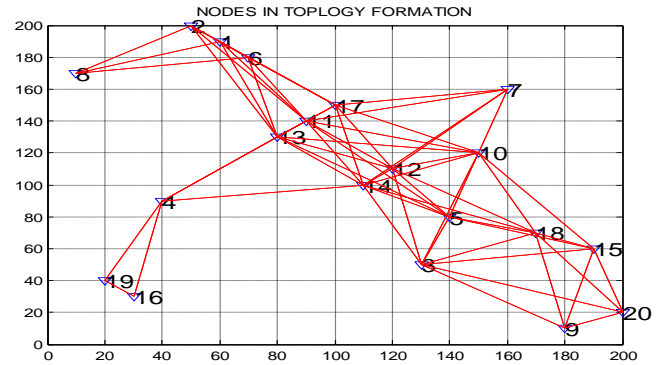

(a)

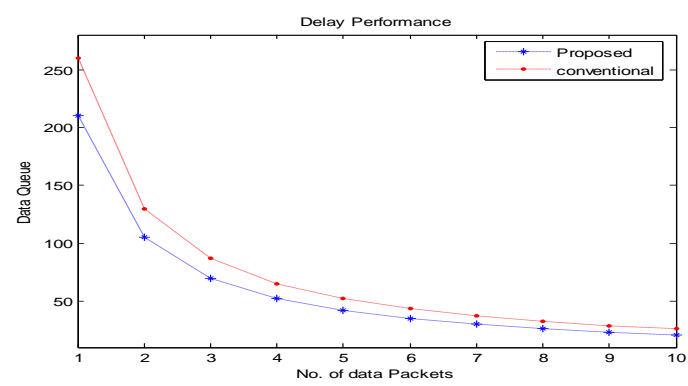

(c)

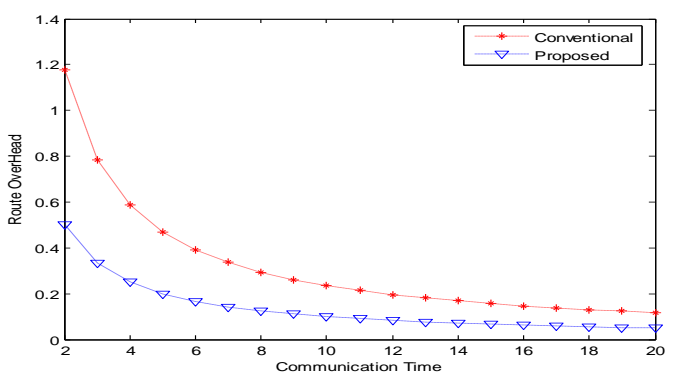

(e)

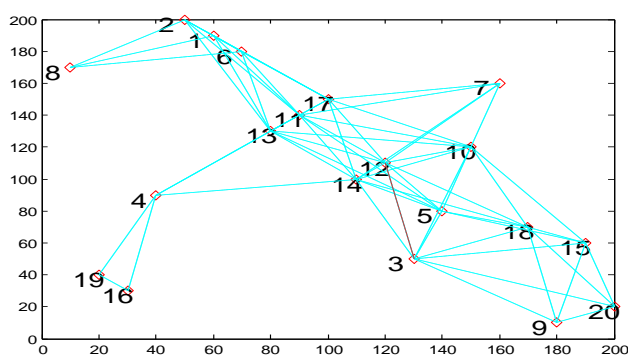

(b)

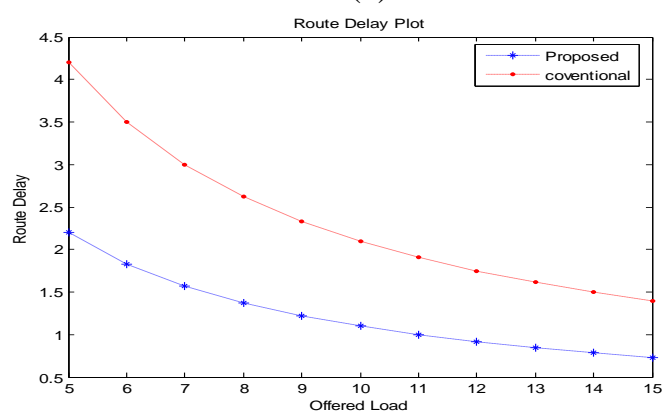

(d)

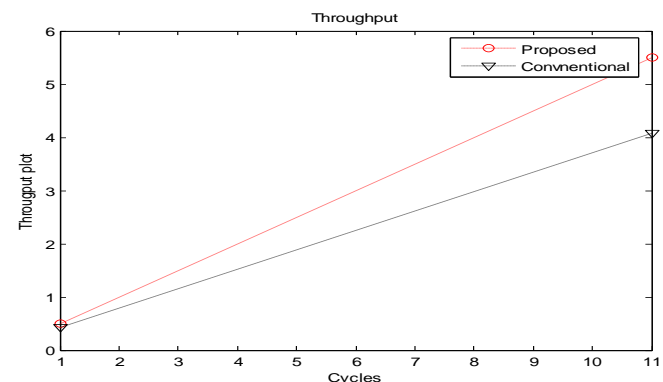

(f)

Figure 7Random topology (a) Nodes in topology (b) Reliable Route (c) Delay Performance (d) Route Delay Plot (e) Route overhead (f) Throughput

\section{Conclusion}

Spectrum is a very valuable resource in wireless communication systems. Cognitive radio, which is one of the efforts to utilize the available spectrum more efficiently through opportunistic spectrum usage, has become an exciting and promising concept. One of the important elements of cognitive radio is sensing the available spectrum opportunities. In this paper by forming an information centric table at the main switching center the idle spectrum of primary users is been allocated to unlicensed users without any interference and throughput is also improvised. This is done by considering two case studies viz., static and adaptive allocation strategies.

\section{References}

[1] I. Mitola, J. and J. Maguire, G. Q., "Cognitive radio: making softwareradios more personal," IEEE Personal Commun. Mag., vol. 6, no. 4,pp. 13-18, Aug. 1999.

[2] Y. Zhao, L. Morales, J. Gaeddert, K. K. Bae, J.-S. Um, and J. H. Reed,“Applying radio environment maps to cognitive wireless regional areanetworks," in Proc. IEEE Int. Symposium on New Frontiers in DynamicSpectrum Access Networks, Dublin, Ireland, Apr. 2007, pp. 115-118.

[3] D. P. Agrawal and Q. A. Zeng, "Introduction to Wireless and Mobile Systems," textbook published by Brooks/Cole, 438 pages, August 2002, ISBN No. 0534-40851-6, second edition April 2005, ISBN No. 0-534-49303-3.

[4] C. Cordeiro and D. P. Agrawal, "Ad hoc \& Sensor Networks, Theory and Applications", World Scientific Publishing, Spring 2006, ISBN No. 81-256-681-3; 81-256-682-1.

[5] Elizabeth M. Royer and C.K. Toh, "A review of current routing protocols for Ad-Hoc Mobile Wireless Networks", IEEE Personal Communications, 46-55, April 1999.

[6] David B. Johnson, "Routing in Ad Hoc Networks of Mobile Hosts", Proceedings of the Workshop on Mobile Computing Systems and Applications, pp. 158163, IEEE Computer Society, Santa Cruz, CA, December 1994.

[7] Perkins, C.E.; Royer, E.M, "Ad-hoc on-demand distance vector routing", Proceedings of Mobile Computing Systems and Applications(HotMobile), 90-100, 1999.

[8] Charles E. Perkins, and PravinBhagwat, "Highly dynamic Destination- Sequenced Distance-Vector routing DSDV for mobile computers", SIGCOMM Comput. Commun. Rev., Vol. 24, Nr. 4 New York, NY, USA:ACM (1994), p. 234-244. 
[9] Ivan Stojmenovic, and Xu Lin, "Loop-Free Hybrid Single-Path/Flooding Routing Algorithms with Guaranteed Delivery for Wireless Networks", IEEE Transactions on Parallel and Distributed Systems, Vol. 12, Nr. 10 (2001), p. 1023-1032.

[10] AnanthRao, Christos H. Papadimitriou, Scott Shenker, and Ion Stoica, "Geographic routing without location information", Proceedings of International Conference on Mobile Computing and Networking(MOBICOMM), 2003, p. 96-108.

[11] Jiangwei Zhou, Yu Chen, Ben Leong, and PratibhaSundarSundaramoorthy, "Practical 3D Geographic Routing for Wireless Sensor Networks", Proceedings of the 8th ACM Conference on Embedded Networked Sensor Systems (SenSys 2010). Zurich, Switzerland. November 2010.

[12] Z.J. Haas and M.R. Pearlman, "The performance of query control schemes for the zone routing protocol", IEEE/ACM Transactions on Networking, Vol. 9, Nr. 4 Piscataway, NJ, USA: IEEE Press (2001), p. 427-438.

[13] Eli M. Gafni and DimitriBertsekas, "Distributed Algorithms for Generating Loop-Free Routes in Networks with Frequently Changing Topology “, IEEE Transactions on Communications, Vol29., No.1, January 1981.

[14] Vincent D. Park and M. Scott Corson, "A Highly Adaptive Distributed Routing Algorithm for Mobile Wireless Networks", Proceedings of the IEEE INFOCOMM 1997, 1405-1413.

[15] P.Bergamo, A. Giovanardi, A. Travasoni, D. Maniezzo, G. Mazzini, and M. Zorzi, "Distributed Power Control for Energy Efficient Routing in Ad Hoc Networks", in Wireless Networks, Vol. 10, Nr. 1 (2004), p. 29-42.

[16] D. Cabric, A. Tkachenko, and R. Brodersen, "Spectrum sensingmeasurements of pilot, energy, and collaborative detection," in Proc.IEEE Military Commun. Conf., Washington, D.C., USA, Oct. 2006,pp. 1-7.

[17] D. Cabric, S. Mishra, and R. Brodersen, "Implementation issues inspectrum sensing for cognitive radios," in Proc. Asilomar Conf. onSignals, Systems and Computers, vol. 1, Pacific Grove, California,USA, Nov. 2004, pp. 772-776.

[18] A. Ghasemi and E. Sousa, "Optimization of spectrum sensing foropportunistic spectrum access in cognitive radio networks," in Proc.IEEE Consumer Commun.and Networking Conf., Las Vegas, Nevada,USA, Jan. 2007, pp. 1022-1026.

[19] N. Khambekar, L. Dong, and V. Chaudhary, "Utilizing OFDM guardinterval for spectrum sensing," in Proc. IEEE Wireless Commun.AndNetworking Conf., Hong Kong, Mar. 2007, pp. 38-42.

[20] S. Geirhofer, L. Tong, and B. Sadler, "A measurement-based model fordynamic spectrum access in WLAN channels," in Proc. IEEE MilitaryCommun. Conf., Washington, D.C., USA, Oct. 2006.

[21] P. Papadimitratos, S. Sankaranarayanan, and A. Mishra, "A bandwidthsharing approach to improve licensed spectrum utilization," IEEECommun. Mag., vol. 43, no. 12, pp. 10-14, Dec. 2005. 